I BIENAL LATINOAMERICANA DIDH

\title{
Derecho ambiental y derecho internacional de los derechos humanos: Impactos en la comprensión y protección del medio ambiente
}

\author{
International human rights and environmental law: Their impact \\ on the understanding and protection of the environment
}

\section{Liliana Galdámez Zelada (iD \\ Universidad de Chile}

\begin{abstract}
RESUMEN Este trabajo analiza la relación entre el derecho internacional de los derechos humanos y el ordenamiento interno, y su especial incidencia en la protección del medio ambiente a partir del trabajo de la Corte Interamericana de Derechos Humanos.
\end{abstract}

PALABRAS CLAVE Incidencia del DIDH en el derecho interno, cláusulas apertura constitucional, comprensión del medio ambiente, Corte Interamericana de Derechos Humanos.

ABSTRACT This work analyzes the relationship between the International Human Rights Law and the domestic system, and its special impact on the protection of the environment based on the work of the Inter-American Court of Human Rights.

KEYWORDS Incidence of IHRL on domestic law, Constitutional opening clauses to IHLR; Understanding the environment, Inter-American Court of Human Rights.

\section{Introducción}

Este trabajo desarrollará en una primera parte breves reflexiones sobre la relación entre el derecho internacional de los derechos humanos en el ordenamiento interno desde una perspectiva regional. En la segunda, analizará el asunto a partir de la configuración de esta relación en la Constitución de Chile. En un tercer apartado, se dará especial atención a la forma en que los criterios de la Corte IDH en materia ambiental 
son compartidos y desarrollados en la jurisprudencia nacional y su incidencia en la comprensión del medio ambiente y carácter sistémico.

\section{La relación entre Constitución y derechos humanos en la región latinoamericana}

Existe una influencia que ha sido determinante en el tratamiento de los derechos fundamentales y su evolución, relacionada con una tendencia en América Latina favorable al reconocimiento del derecho internacional en el derecho interno. Estos criterios han sido especialmente decisivos para el fortalecimiento del derecho a la tutela judicial efectiva en casos de graves violaciones a los derechos humanos, y han permitido abordar otros problemas desafiantes en el actual contexto, como los ambientales y sus efectos en el derecho a la salud.

La relación entre el derecho internacional de los derechos humanos con el ordenamiento interno ha sido clave para avanzar en desarrollos favorables a la protección de nuevos derechos o niveles más altos de protección en los derechos contenidos en la Constitución (von Bogdandy, 2015, 2017). A diferencia de lo que ocurre en Chile, la mayoría de los países latinoamericanos que han mostrado una mayor apertura constitucional al derecho internacional cuentan con una Carta que ha sido el resultado de procesos constituyentes democráticos, no asimilables al caso chileno. Es quizá esta misma cuestión la que ha impulsado una evolución tan novedosa y constante en la aplicación e interpretación del artículo 5 de la Constitución chilena, que contiene la cláusula de apertura constitucional al derecho internacional de los derechos humanos.

En el ámbito latinoamericano, todas las constituciones han admitido, con mayor o menor amplitud y claridad, la recepción del derecho internacional en el derecho interno, a cuya delimitación también han contribuido cortes y salas constitucionales. Al valorar este reconocimiento, por amplio o restringido que sea, es oportuno recordar la afirmación de Bobbio (1991: 63): «El problema grave de nuestro tiempo respecto a los derechos humanos no [es] el de fundamentarlos, sino el de protegerlos». Este reconocimiento en muchos países de la región no es garantía de su protección.

La mayoría de las constituciones vigentes en América Latina se gestaron en la segunda mitad del siglo XX, después de conflictos armados internos y dictaduras militares. ${ }^{1}$ Surgidas del consenso por la búsqueda de democracia, todas ellas se mani-

1. Constitución de Colombia de 1991, con Gobierno militar entre 1957 y 1958; Constitución de Argentina de 1853, su última reforma es de 1994, con Gobierno militar entre 1976 y 1983; Constitución de Perú de 1993, con Gobierno militar entre 1968 y 1975; Constitución de Guatemala de 1985 y reforma constitucional en 1993, con conflicto armado interno entre 1960 y 1996; Constitución de El Salvador de 1983, 
fiestan en favor de la consecución del bien común, el desarrollo y el reconocimiento de la dignidad de la persona. ${ }^{2}$

Este trabajo hará referencia exclusivamente a normas contenidas en tratados en relación con derechos humanos. No se analizará la posición de todo el derecho internacional en el derecho interno, pues existe una tendencia a separar el tratamiento del derecho internacional general del derecho internacional de los derechos humanos, derecho internacional humanitario y derecho penal internacional. La doctrina coincide en señalar que este tipo de tratados posee características específicas que lo distinguen de otros. Así lo ha dicho la Corte Interamericana en su Opinión Consultiva 2-82, del 24 de septiembre de 1982 (número 29):

Los tratados modernos sobre derechos humanos en general, y en particular la Convención Americana, no son tratados multilaterales de tipo tradicional, concluidos en función de un intercambio recíproco de derechos, para el beneficio mutuo de los Estados contratantes. Su objeto y fin son la protección de los derechos fundamentales de los seres humanos, independientemente de su nacionalidad, tanto frente a su propio Estado como frente a los otros Estados contratantes. Al aprobar estos tratados sobre derechos humanos, los Estados se someten a un orden legal dentro del cual ellos, por el bien común, asumen varias obligaciones, no en relación con otros Estados, sino hacia los individuos bajo su jurisdicción. El carácter especial de estos tratados ha sido reconocido, entre otros, por la Comisión Europea de Derechos Humanos cuando declaró que las obligaciones asumidas por las Altas Partes Contratantes en la Convención [Europea] son esencialmente de carácter objetivo, diseñadas para proteger los derechos fundamentales de los seres humanos de violaciones de parte de las altas partes contratantes en vez de crear derechos subjetivos y recíprocos entre las altas partes.

con gobierno militar entre 1931 y 1979; Constitución de Bolivia de 1967, con Gobierno militar entre 1971 y 1978 (el 22 de octubre de 2008 se aprobó una nueva Constitución); Constitución de Honduras de 1982, con Gobierno militar entre 1972 y 1982; Constitución de Paraguay de 1992, con Gobierno militar entre 1954 y 1989; Constitución de Brasil de 1988, con Gobierno militar entre 1964 y 1985.

2. Por ejemplo, la Constitución de Guatemala, preámbulo: «Nosotros, los representantes del pueblo de Guatemala, electos libre y democráticamente, reunidos en Asamblea Nacional Constituyente, con el fin de organizar jurídica y políticamente el Estado; afirmando la primacía de la persona humana como sujeto y fin del orden social»; Constitución de Perú, artículo 1: «La defensa de la persona humana y el respeto de su dignidad son el fin supremo de la sociedad y del Estado»; y Constitución de Colombia, artículo 1: «Colombia es un Estado social de derecho, organizado en forma de república unitaria, descentralizada, con autonomía de sus entidades territoriales, democrática, participativa y pluralista, fundada en el respeto de la dignidad humana, en el trabajo y la solidaridad de las personas que la integran y en la prevalencia del interés general». 


\section{La relación entre el derecho internacional de derechos humanos y el ordenamiento interno: La experiencia de Chile}

El artículo 5 inciso segundo de la Constitución chilena, mediante reforma constitucional en 1989, introduce la siguiente reforma al texto: «Es deber de los órganos del Estado respetar y promover tales derechos, garantizados por esta Constitución, así como por los tratados internacionales ratificados por Chile y que se encuentren vigentes».

Desde los orígenes de la norma tuvo lugar una activa polémica, retratada en la revista Ius Et Praxis, año 2, número 2, de 2003, que dedica este número a reunir distintas tesis sobre este debate, en la que se sostienen opiniones a favor del valor constitucional de los tratados sobre derechos humanos, en una interpretación primera del inciso segundo del artículo 5 de la Constitución, y otras en pro de la tesis que sostiene su posición infraconstitucional, mientras que una tercera tesis sostiene que el sentido del artículo 5 es reforzar los derechos esenciales que emanan de la naturaleza humana (Ríos, 1998).

En la actualidad, las tesis se van aproximando y logrando un mayor consenso en torno a la idea de los derechos esenciales que emanan de la naturaleza humana contenidos en tratados internacionales sobre derechos humanos como fuente material de la Constitución. Se puede afirmar en este sentido que, a más de 30 años de la reforma a la Constitución, los derechos humanos contenidos en tratados ratificados y vigentes contribuyen a completar el catálogo de derechos que la Constitución contempla, integrándose a ella. Es la jurisprudencia de la Corte Suprema y Cortes de Apelaciones la que con mayor intensidad ha contribuido a reforzar esta interpretación.

Se puede afirmar que hoy el debate a propósito de la jerarquía de los tratados no está en el centro de la polémica. En general, la jurisprudencia ha hecho una interpretación conforme al derecho internacional de los derechos humanos, desplazando normas nacionales o reinterpretándolas a la luz de los compromisos asumidos por el país a partir de la ratificación de tratados internacionales sobre derechos humanos. ${ }^{3}$ No es una cuestión de jerarquía, sino que la propia Constitución conecta los derechos fundamentales con los derechos de fuente internacional.

Por otra parte, diversas cuestiones de orden procesal han impulsado otros desarrollos interesantes a partir de la referencia al derecho internacional de los derechos humanos en la Constitución. Uno de sus ámbitos de incidencia han sido los derechos

3. Véase Comunidad indígena Entuco con Municipalidad de Padre Las Casas, Corte de Apelaciones de Temuco, 20 de noviembre de 2015; Comunidad indígena Entuco con Contreras Sepúlveda y otros, Corte Suprema, rol 17037-13, delitos de lesa humanidad, prescripción penal y civil, 8 de octubre de 2014, cita MJCH_MJ39074; Fries Monleon, Lorena y otros con Gendarmería de Chile, Corte de Apelaciones de Antofagasta, rol 68-13, recurso de amparo, 7 de diciembre de 2013. 
fundamentales, por lo que, a la hora de alegar la vulneración de derechos humanos que no se contienen en el texto de la Constitución, o bien, de derechos fundamentales contenidos en la Constitución cuya privación, perturbación o amenaza no permita recurrir vía recurso de protección para su tutela, se ha desarrollado otra tendencia que merece la pena destacar. En ambos supuestos, es usual que para alegar la vulneración de derechos que no aparecen en forma expresa en la Constitución o derechos que no contemplen recurso de protección para su defensa, en los alegatos y conclusiones de los jueces adquiera relevancia el artículo 1 de la Constitución: «Las personas nacen libres e iguales en dignidad y derechos», asociado a algún derecho que sí esté tutelado por el recurso de protección, principalmente, igualdad (artículo 19 número 2) o propiedad (artículo 19 número 24). A estos argumentos se suma un tercero: que el derecho cuya protección se impulsa está contenido en un tratado internacional ratificado y vigente.

El derecho internacional de los derechos humanos ha traído un dinamismo insospechado en la comprensión de los derechos fundamentales, y permitido el dinamismo y desarrollo progresivo del catálogo de derechos. Ahora bien, esta tarea no reemplaza la del legislador para el desarrollo de los derechos fundamentales. Estos progresos en ocasiones impulsan la tarea del legislador; en otras, propician el desarrollo jurisprudencial de los derechos caso a caso, lo que implica un desarrollo jurisprudencial que no se concreta necesariamente para quienes no litigan ante las cortes superiores.

En la actualidad existe un ámbito consolidado de incidencia del derecho internacional de los derechos humanos en el ordenamiento nacional, que además configura un derecho común en la región. Se trata de los criterios utilizados en el enjuiciamiento y sanción de causas por graves violaciones a los derechos humanos.

El deber de prevenir, juzgar, sancionar y reparar a las víctimas en estos casos ha sido una de las primeras materias impulsadas por la Corte IDH a partir del caso Velásquez Rodríguez con Honduras. Otra de las sentencias importantes en este ámbito ha sido el caso Almonacid Arellano con Chile, en el que la Corte ordenó al Estado chileno remover los obstáculos que impiden la investigación de los hechos que dieron lugar a la muerte del profesor Almonacid en 1973. A partir de esta sentencia, la Corte impulsó de manera sistemática el llamado control de convencionalidad y se consolidó la inaplicabilidad de las leyes de amnistía en causas por graves violaciones a los derechos humanos en varios países de la región: Argentina, Uruguay, Perú y Chile, entre otros. De la misma manera ocurrió con la prescripción de la acción penal y más adelante con la prescripción de la acción civil.

Pero el ámbito de incidencia del derecho internacional de los derechos humanos - y también del derecho comparado- en el ordenamiento nacional se ha extendido en la actualidad a otros asuntos relacionados con los derechos fundamentales: el desarrollo progresivo de la jurisprudencia de la Corte IDH de los derechos econó- 
micos, sociales, culturales y ambientales. La incidencia del derecho internacional de los derechos humanos en esos derechos debilitados en el texto de la Constitución, y fortalecidos en la jurisprudencia de los tribunales y por el legislador, es un proceso paralelo y sincrónico a lo que ocurre en el ámbito interamericano. Sentencia clave en esta materia es la del caso Poblete Vilches con Chile, a propósito del acceso a la salud de una persona mayor, en que el mismo esfuerzo argumentativo que desarrolla la Corte para la protección del derecho a la salud, a partir del artículo 26 de la Convención Americana sobre Derechos Humanos (CADH), se ha desarrollado por los jueces nacionales que asocian salud, vida y en algunos casos derecho de propiedad, a fin de asegurar una dimensión del derecho a la salud que no protege el recurso de protección.

En materia medioambiental se aprecia otro fenómeno semejante al descrito, quizá mucho más complejo y profundo por la propia naturaleza de lo ambiental. Se trata de una transformación y evolución en la comprensión del medio ambiente, su interconexión con otros derechos fundamentales, principios de interpretación e incluso la superación de una mirada estrictamente antropocéntrica en la relación entre ser humano y naturaleza. Estos desarrollos, es cierto, no siempre aparecen conectados de forma expresa: en el caso al que me referiré en el siguiente apartado, los jueces nacionales no citan a la Corte IDH; sin embargo, la forma en que se comprende lo ambiental, las relaciones con la vida, la economía y los principios ambientales de interpretación se configuran de forma sincrónica. Se trata de desarrollos y evoluciones en que convergen ordenamiento nacional e interamericano.

A estos efectos, quisiera en el siguiente apartado analizar la Opinión Consultiva OC-23/17 de la Corte IDH, del 15 de noviembre de $2017,{ }^{4}$ y la sentencia dictada por

4. Opinión Consultiva OC-23/17, Corte Interamericana de Derechos Humanos, 15 de noviembre de 2017, solicitada por la República de Colombia, medio ambiente y derechos humanos. El 14 de marzo de 2016, la República de Colombia, con fundamento en el artículo 64.11 de la Convención Americana y de conformidad con lo establecido en el artículo 70.1 y 70.22 del Reglamento, presentó una solicitud de opinión consultiva sobre las obligaciones de los Estados en relación con el medio ambiente en el marco de la protección y garantía de los derechos a la vida y a la integridad personal, a fin de que el Tribunal determine «de qué forma se debe interpretar el Pacto de San José cuando existe el riesgo de que la construcción y el uso de las nuevas grandes obras de infraestructura afecten de forma grave el medio ambiente marino en la región del Gran Caribe y, en consecuencia, el hábitat humano esencial para el pleno goce y ejercicio de los derechos de los habitantes de las costas y/o islas de un Estado parte del Pacto, a la luz de las normas ambientales consagradas en tratados y en el derecho internacional consuetudinario aplicable entre los Estados respectivos». Asimismo, el Estado solicitante buscaba que la Corte determine «cómo se debe interpretar el Pacto de San José en relación con otros tratados en materia ambiental que buscan proteger zonas específicas, como es el caso del Convenio para la Protección y el Desarrollo del Medio Marino en la región del Gran Caribe, con relación a la construcción de grandes obras de infraestructura en Estados parte de estos tratados y las respectivas obligaciones internacionales en materia de prevención, precaución, mitigación del daño y de cooperación entre los Estados que se pueden ver afectados». 
la Corte Suprema en el caso Quintero Puchuncaví de 2019. ${ }^{5}$ Como se podrá observar, elementos comunes convergen para una nueva comprensión del medio ambiente, relevante para el caso chileno, en el que se evidencia una creciente conflictividad socioambiental.

\section{La incidencia del derecho internacional de los derechos humanos en el tratamiento sistémico del medio ambiente}

Una serie de informes en Chile viene dando cuenta de constantes y complejas tensiones entre el desarrollo de la industria de la minería, de las energías y la afectación de derechos de las comunidades que habitan en las proximidades de esa industria o que se ve afectada por sus externalidades. ${ }^{6}$

Uno de los problemas más críticos que se ha planteado, y que aún persiste, se produce en Chile a propósito del desarrollo industrial de la zona de Quintero y $\mathrm{Pu}$ chuncaví. Una notable sentencia dictada en 2019 por la Corte Suprema analiza los problemas asociados con la industria, el rol del Estado, las afectaciones a las personas en derechos como la vida, la salud e integridad, e incorpora de manera consistente la noción de desarrollo sostenible, que no ha sido invocada de manera sistemática por la jurisdicción ordinaria y la constitucional. Esta sentencia, como señalé, es coherente con los criterios de la Corte IDH sostenidos en la OC 23/17. Analizaré enseguida ambas resoluciones e incluiré un apartado en conclusiones en la parte final de este trabajo.

\section{La Opinión Consultiva OC-23/17}

Conviene dejar establecido que los casos que analizaré a continuación, así como tienen importantes semejanzas desde la perspectiva del problema que analizan, plantean matices también en cuanto a los sujetos afectados en su derecho a vivir en un medio ambiente sano. La opinión consultiva hace referencia a uno de los actores involucrados en la decisión, esto es, los pueblos indígenas y tribales, quienes se verían afectados en sus derechos ambientales; mientras que para el caso Quintero Puchuncaví, los derechos afectados son de poblaciones que padecen las externalidades de un complejo industrial que no responde a la identidad de los pueblos indígenas. En ambos casos las cuestiones analizadas comprometen a comunidades y poblaciones como sujetos colectivos, por lo que las reflexiones respecto de la afectación de sus derechos tienen importantes semejanzas.

5. Sentencia del caso Quintero Puchuncaví, Corte Suprema de Chile, Tercera Sala, rol 5888-201, recurso de protección, 28 de mayo de 2019.

6. Véase el «Mapa de conflictos socioambientales», Instituto Nacional de Derechos Humanos, disponible en https://mapaconflictos.indh.cl/. 
Para el análisis de ambos casos, he identificado ciertos criterios a la hora de abordar cada uno. Por esta razón, las ideas serán sistematizadas a partir de cinco criterios. Iniciaré la reflexión con el análisis de la OC-23/17, anterior a la sentencia del caso Quintero Puchuncaví.

\section{La configuración del derecho a un medio ambiente sano y sus fuentes}

En la Opinión Consultiva OC-23/17, la Corte deja claro ya desde sus inicios que «no se encuentra limitada por los términos literales de las consultas que se le plantean para el ejercicio de su función consultiva». En esta Opinión, la Corte explica que, para la configuración del derecho a un medio ambiente sano en el Sistema Interamericano de Derechos Humanos, invocará no solo la Convención, sino que un conjunto de otras fuentes del derecho internacional, junto al principio pro persona, la interpretación evolutiva de los tratados internacionales sobre derechos humanos y otros criterios destacados. Con ello busca, en definitiva, establecer que los derechos de contenido ambiental también forman parte de los derechos del Sistema Interamericano.

En esta Opinión, la Corte reitera la idea de una interpretación integradora de todas las fuentes del derecho, nacionales e internacionales:

Es en este sentido que la Convención Americana prevé expresamente determinadas pautas de interpretación en su artículo 29, entre las que alberga el principio pro persona, que implican que ninguna disposición de dicho tratado puede ser interpretada en el sentido de limitar el goce y ejercicio de cualquier derecho o libertad que pueda estar reconocido de acuerdo con las leyes de cualquiera de los Estados parte o de acuerdo con otra convención en que sea parte uno de dichos Estados, o bien de excluir o limitar el efecto que puedan producir la Declaración Americana de los Derechos y Deberes del Hombre y otros actos internacionales de la misma naturaleza.

Sobre el sistema de fuentes,

la Corte desea subrayar que, aunque no le corresponde emitir una interpretación directa de los distintos instrumentos de derecho ambiental, indudablemente los principios, derechos y obligaciones allí contenidos contribuyen en forma decisiva a fijar el alcance de la Convención Americana. En virtud de la materia sometida a consulta, la Corte tendrá en consideración, como fuentes de derecho internacional adicionales, otras convenciones relevantes a fin de efectuar una interpretación armónica de las obligaciones internacionales en los términos de la disposición citada. En adición, la Corte considerará las obligaciones aplicables y la jurisprudencia y decisiones al respecto, así como las resoluciones, pronunciamientos y declaraciones referentes al tema que hubieren sido adoptados a nivel internacional (el énfasis es nuestro). 


\section{La interrelación entre los derechos humanos y el medio ambiente}

En este ámbito afirma la Corte que ha «reconocido la existencia de una relación innegable entre la protección del medio ambiente y la realización de otros derechos humanos, en tanto la degradación ambiental y los efectos adversos del cambio climático afectan el goce efectivo de los derechos humanos». La Corte resalta lo que ha señalado la Asamblea General de la OEA, cuando dice que «ha reconocido la estrecha relación entre la protección al medio ambiente y los derechos humanos [...] y destacado que el cambio climático produce efectos adversos en el disfrute de los derechos humanos». Destaca en el mismo sentido las opiniones de otros sistemas regionales de protección a los derechos humanos:

En el ámbito europeo, el Tribunal Europeo de Derechos Humanos ha reconocido que la degradación severa del medio ambiente puede afectar el bienestar del individuo y, como consecuencia, generar violaciones a los derechos de las personas, tales como los derechos a la vida, al respeto a la vida privada y familiar y a la propiedad privada. De manera similar, la Comisión Africana de Derechos Humanos y de los Pueblos ha indicado que el derecho a un «medio ambiente general satisfactorio, favorable al desarrollo» está estrechamente relacionado con los derechos económicos y sociales en la medida en que el medio ambiente afecta la calidad de vida y la seguridad del individuo.

\section{Protección del medio ambiente y desarrollo sostenible}

La Corte introduce por vía interpretativa la noción de desarrollo sostenible, que permite articular y relacionar el desarrollo de la industria con el respeto a los derechos humanos. Este principio no encuentra un reconocimiento expreso en la $\mathrm{CADH}$, de la misma manera que tampoco lo incorpora la Constitución chilena de los ochenta. En ambos casos este criterio se introduce por vía interpretativa. Sobre este punto, la Opinión señala:

Existe un amplio reconocimiento en el derecho internacional sobre la relación interdependiente entre la protección al medio ambiente, el desarrollo sostenible y los derechos humanos. Dicha interrelación se ha afirmado desde la Declaración de Estocolmo sobre el Medio Ambiente Humano, donde se estableció que «[e]l desarrollo económico y social es indispensable para asegurar al hombre un ambiente de vida y trabajo favorable y crear en la Tierra las condiciones necesarias para mejorar la calidad de la vida», afirmándose la necesidad de balancear el desarrollo con la protección del medio humano. Posteriormente, en la Declaración de Río sobre el Medio Ambiente y el Desarrollo, los Estados reconocieron que «[1]os seres humanos constituyen el centro de las preocupaciones relacionadas con el desarrollo sostenible» $y$, 
a la vez, destacaron que «a fin de alcanzar el desarrollo sostenible, la protección del medio ambiente deberá constituir parte integrante del proceso de desarrollo».

\section{Principio precautorio y preventivo, y procedimentales}

Para el cumplimiento de las obligaciones de respetar y garantizar los derechos a la vida y a la integridad personal, en el contexto de la protección del medio ambiente, los Estados deben cumplir con una serie de obligaciones, tanto para daños ocurridos dentro de su territorio como para daños que traspasen sus fronteras [...] a) la obligación de prevención; b) el principio de precaución; c) la obligación de cooperación, y d) las obligaciones procedimentales en materia de protección del medio ambiente, con el propósito de establecer y determinar las obligaciones estatales derivados de la interpretación sistemática de dichas normas junto con las obligaciones de respetar y garantizar los derechos a la vida e integridad personal consagrados en la Convención Americana.

\section{La vulnerabilidad}

La Corte toma en cuenta que la afectación a estos derechos puede darse con mayor intensidad en determinados grupos en situación de vulnerabilidad. Se ha reconocido que los daños ambientales «se dejarán sentir con más fuerza en los sectores de la población que ya se encuentran en situaciones vulnerables», por lo cual, con base en «la normativa internacional de derechos humanos, los Estados están jurídicamente obligados a hacer frente a esas vulnerabilidades, de conformidad con el principio de igualdad y no discriminación».

\section{Caso Quintero Puchuncaví}

La Constitución chilena de 1980 ha tratado de forma separada la protección de la salud y el medio ambiente a partir de la configuración de dos derechos diferenciados. La forma de lo ambiental en la Constitución carece de una mirada desde la teoría de los sistemas, no contempla la interconexión entre salud, medio ambiente, vida e integridad y carece de mecanismos auxiliares de interpretación en materia ambiental (Galdámez, 2017, 2019, 2020). También da un tratamiento separado a un elemento esencial de la naturaleza: el agua. Sin embargo, a partir de los desarrollos del derecho internacional, de las opiniones de la Corte IDH y de informes y opiniones de órganos del sistema universal y regionales de protección de los derechos humanos, la jurisprudencia nacional ha desarrollado las dimensiones sistémicas del medio ambiente, la idea de su interconexión, en armonía con los desarrollos de lo ambiental en el derecho internacional. 
En el caso denominado Quintero Puchuncaví, por episodios reiterados de contaminación en una zona que paradojalmente se conoce como «zona de sacrificio ambiental», la Corte Suprema recogió múltiples desarrollos elaborados en el ámbito del derecho internacional ambiental y de derechos humanos para la comprensión del medio ambiente. Cabe destacar que se trata de una sentencia valiosa, pero que falta aún examinar su posterior continuidad en otros casos semejantes.

\section{La configuración del derecho a un medio ambiente sano y sus fuentes}

Respecto de la importancia que adquiere el derecho internacional en materia ambiental, la sentencia resalta:

Que, asimismo, cabe subrayar que el artículo 70 de la Ley 19.300 prescribe, en lo que interesa, que: «Corresponderá especialmente al Ministerio: [...] d) Velar por el cumplimiento de las convenciones internacionales, en que Chile sea parte en materia ambiental, y ejercer la calidad de contraparte administrativa, científica o técnica de tales convenciones, sin perjuicio de las facultades del Ministerio de Relaciones Exteriores».

En esta materia, los jueces constatan que el Ministerio del Medio Ambiente chileno

ha dejado de aplicar al caso en estudio distintos instrumentos internacionales, entre los que se menciona el Protocolo de Montreal, relativo a las sustancias agotadoras de la capa de ozono, que fuera promulgado mediante el Decreto Supremo 238 de 1990 del Ministerio de Relaciones Exteriores; el Convenio de Estocolmo sobre Contaminantes Orgánicos Persistentes, promulgado a través del Decreto Supremo 38 de 2005 del Ministerio de Relaciones Exteriores, y el Convenio de Basilea relativo al control de los movimientos transfronterizos de desechos peligrosos y su eliminación, promulgado mediante el Decreto Supremo 685 de 1992 del Ministerio de Relaciones Exteriores. Todas, como se evidencia, normas aplicables y exigibles en Chile.

\section{La interrelación entre los derechos humanos y el medio ambiente}

Señala la Corte Suprema:

En la situación general de grave contaminación [...] se entrelazan posibles afectaciones a tres derechos fundamentales, en particular la vida de las personas, su salud y su derecho a vivir en un medio ambiente libre de contaminación, siendo pertinente analizar su eventual vulneración en conjunto, puesto que una afectación seria de la salud, por causa de una emergencia ambiental severa, amenaza también la vida y, en todo caso, la integridad física o, cuando menos, psíquica, de las personas. 


\section{Protección del medio ambiente y desarrollo sustentable}

La sentencia incorpora la noción de desarrollo sustentable, que no contempla la Constitución, pero que los jueces hacen suya a partir de diversas declaraciones internacionales en la materia. Considera la sentencia que la noción de desarrollo sustentable se asocia a "equidad intergeneracional", la "explotación apropiada o racional", el "uso equitativo de los recursos naturales y utilización por otros Estados" y la "integración del medio ambiente al desarrollo"».

Agrega que no se ha respetado ni cumplido la noción de desarrollo sustentable:

En otras palabras, la actividad de los agentes económicos asentados en la zona de Quintero, Ventanas y Puchuncaví no solo se ha llevado a efecto sin implementar medidas «apropiadas de conservación y protección del medio ambiente», sino que, por el contrario, ha supuesto una importante fuente de contaminación para el entorno de esas comunas, generando episodios de intoxicación cuya última expresión está constituida por aquellos acaecidos los días 21 y 23 de agosto y 4 de septiembre de 2018. Tal constatación representa una clara y evidente transgresión del concepto de desarrollo sustentable reconocido en nuestro derecho interno, así como por los tratados y convenciones internacionales que rigen esta materia.

\section{Principios interpretativos precautorio y preventivo y procedimentales}

Resulta relevante la anotada falta de elementos de juicio para determinar tanto las causas como los efectos precisos de los episodios de contaminación objeto de los recursos, pues debido a ella este tribunal deberá recurrir como elementos orientadores de su proceder a dos principios de la mayor trascendencia en el ámbito del derecho ambiental, cuales son el precautorio y el de prevención. En torno al primero se ha dicho que «el principio precautorio impone una actuación anticipada, incluyendo las situaciones en que no se cuenta con la certeza absoluta de los efectos que un determinado hecho puede tener para el medio ambiente», mientras que el segundo, esto es, el principio de prevención, «supone el conocimiento científico de las consecuencias ambientales de una determinada actividad. Es decir, opera cuando el daño ambiental es previsible, de acuerdo con la evidencia con que se cuenta. El ámbito de aplicación del principio precautorio, en cambio, es una etapa anterior: opera en casos de una amenaza potencial, pero debido a la incertidumbre o controversia científica no es posible hacer una predicción apropiada del impacto ambiental» [...] Que los jueces de Iberoamérica han asumido el conocimiento y aplicación de los principios jurídicos medioambientales para un desarrollo ecológicamente sustentable, expresando como parte del principio de acceso a la justicia ambiental que las sentencias deben concretar soluciones que constituyan procesos de larga duración (Principio 17, XVII, letra f). En «Principios jurídicos medioambientales para un desarrollo ecológicamente sustentable». Poder Judicial de la República de Chile, 
Organización de Estados Americanos y Cumbre Judicial Iberoamericana [...] es por ello que se deberá tener en cuenta «las consideraciones ecológicas al momento de formular y aplicar las políticas económicas y sectoriales, en los procedimientos y en la toma de decisiones de los poderes públicos, en la dirección y el desarrollo de los procesos de producción y en el comportamiento y elecciones de las autoridades» [...] en ese mismo sentido se ha reconocido que cada «Estado, entidad pública o privada y los particulares tienen la obligación de cuidar y promover el bienestar de la naturaleza, independientemente de su valor para los seres humanos, al igual que de imponer limitaciones a su uso y explotación» [...] que «cada ser humano, presente y futuro, tiene derecho a un medio ambiente sano, seguro, saludable y sostenible» [...] y que el «aprovechamiento sostenible de los recursos naturales y la preservación del patrimonio natural y cultural son condicionantes necesarios del desarrollo económico y social».

\section{La vulnerabilidad}

Que relacionado con lo anterior, se debe consignar que los menores de edad no constituyen la única población vulnerable y particularmente expuesta a la deteriorada calidad del ambiente que se vive en Quintero, Ventanas y Puchuncaví, pues existen, también, verbi gracia, niños y niñas más jóvenes que, por su edad, aún no ingresan al sistema escolar; además, están los ancianos y las personas enfermas, cuya condición de salud se puede ver especialmente afectada por la indicada contaminación, y las mujeres embarazadas. Respecto de ellas, y cada vez que se produzca un evento crítico de contaminación, la autoridad local, asesorada y apoyada, si es necesario, por los niveles provincial y regional, deberá disponer lo pertinente para sacar del sector perjudicado por tal circunstancia a toda la población vulnerable hacia lugares seguros y mientras perdure el señalado episodio.

\section{Conclusiones}

El derecho internacional, tanto de los derechos humanos como ambiental, ha aportado a la evolución del tratamiento del medio ambiente en los ordenamientos internos. Sus desarrollos convencionales han incidido en los desarrollos normativos internos y también en los criterios aplicados por la jurisprudencia.

Los aportes provenientes de la Corte IDH adquieren dimensiones propias cuando hablamos de medio ambiente. No se trata, como en el caso de graves violaciones a los derechos humanos, de desplazar o inaplicar instituciones asociadas a la impunidad como la amnistía y la prescripción. En materia ambiental, los criterios internacionales han propiciado una nueva forma de comprensión del medio ambiente. El juez nacional e interamericano concluyen en el mismo sentido que las afectaciones del 
medio ambiente tienen consecuencias en otros derechos humanos; eso implica que sus decisiones sean también más complejas.

A partir del derecho internacional, los jueces incorporarán por vía interpretativa la idea del desarrollo sustentable como bisagra para resolver las tensiones entre desarrollo y protección de los derechos humanos y fundamentales. No se trata de un principio que tenga consagración convencional ni constitucional expresa - en el caso de Chile-; sin embargo, se va configurando como una de las claves a la hora de abordar las tensiones y los límites que debe observar la industria. Para el caso chileno, la sentencia del caso Quintero Puchuncaví deja instalada una expectativa: que el desarrollo sustentable se consolide como criterio de los jueces. Es de esperar que en esta dirección evolucione la jurisprudencia del Tribunal Constitucional, muy lejana aún de estos criterios.

Reglas de interpretación como el principio preventivo, precautorio y derechos procedimentales, comienzan también a formar parte de una nueva comprensión de lo ambiental, lo que da cuenta de la necesidad de tener reglas especiales para abordar problemas complejos. Se espera que el principio precautorio logre consolidarse como criterio central para abordar lo ambiental. Lo mismo la valiosa invocación de la vulnerabilidad, para efectivamente proteger a quienes están expuestos con mayor intensidad al riesgo ambiental.

La configuración de un corpus iuris del medio ambiente, al que concurre un conjunto de fuentes nacionales e internacionales, es otro de los asuntos que ambas sentencias comparten. Los desafíos ambientales, muchos de naturaleza transfronteriza, requieren respuestas globales e integradas. En el caso de la sentencia Quintero $\mathrm{Pu}$ chuncaví, es un avance que el juez nacional atribuya y concrete los deberes de control y protección que pesan sobre el Estado para velar por un efectivo cumplimiento de los compromisos que asume en el ámbito internacional. Si esta tendencia logra consolidarse, contaremos con criterios comunes entre los Estados y los sistemas internacionales de protección de los derechos humanos. Esto permitirá avanzar de manera auspiciosa hacia la idea de un derecho común, global, coherente con los desafíos que con tanta fuerza demanda la protección del ambiente y que se hacen aún más evidentes en tiempos de pandemia.

\section{Referencias}

Bоввіо, Norberto (1991). El tiempo de los derechos. Madrid: Sistema.

Galdámez Zelada, Liliana (2017). «Medio ambiente, Constitución y tratados en Chile». Boletín Mexicano de Derecho Comparado, 148: 113-144. DOI: 10.22201/ iij.24484873e.2017.148.10997. 
-. (2018). «Recurso de protección y medio ambiente en la jurisprudencia de la Corte Suprema». En Liliana Galdámez Zelada (coordinadora), Una perspectiva constitucional del medio ambiente. Santiago: Jurídica de Chile.

-. (2019). «El medio ambiente en la jurisprudencia del Tribunal Constitucional de Chile». Revista de la Facultad de Derecho de la Universidad de la República del Uruguay, 48: e107. DOI: 10.22187/ $\mathrm{rfd} 2020 \mathrm{n} 48 \mathrm{a} 7$.

Ríos, Lautaro (1998). «Jerarquía normativa de los tratados internacionales sobre derechos humanos». Gaceta Jurídica, 215: 7-14. Disponible en https://bit.ly/3iJGDb9. Von Bogdandy, Armin (2015). «Ius constitutionale commune en América Latina: Una mirada a un constitucionalismo transformador». Revista de Derecho del Estado, 34: 3-50. DOI: 10.18601/01229893.n34.01.

Von Bogdandy, Armin, Mariela Morales y Eduardo Ferrer Mac-Gregor (2017). Ius constitutionale commune en América Latina: Textos básicos para su comprensión. Querétaro: Instituto de Estudios Constitucionales del Estado de Querétaro y Max Planck Institute for Comparative Public Law and International Law.

\section{Sobre la autora}

Liliana Galdámez Zelada es doctora en Derecho por la Universidad de Valladolid, España. Investigadora en el Centro de Derechos Humanos y la Facultad de Derecho de la Universidad de Chile. Su correo electrónico es lgaldamez@derecho.uchile. cl. (iD) https://orcid.org/0000-0003-2889-1629. 
El Anuario de Derechos Humanos es una publicación semestral de referencia y consulta en materia de derechos humanos y campos afines. Busca ser un espacio de discusión de los temas centrales en el ámbito nacional e internacional sobre derechos humanos. Es publicado desde 2005 por el Centro de Derechos Humanos de la Facultad de Derecho de la Universidad de Chile.

\author{
EDITORA GENERAL \\ Claudia Iriarte Rivas \\ ciriarter@derecho.uchile.cl \\ EDITORA DE ESTE NÚMERO \\ Liliana Galdámez Zelada \\ lgaldamez@derecho.uchile.cl \\ SITIO WEB \\ anuariocdh.uchile.cl \\ CORREO ELECTRÓNICO \\ anuario-cdh@derecho.uchile.cl \\ LICENCIA DE ESTE ARTÍCULO
}

Creative Commons Atribución Compartir Igual 4.o Internacional

\author{
कै \\ La edición de textos, el diseño editorial \\ y la conversión a formatos electrónicos de este artículo \\ estuvieron a cargo de Tipográfica \\ (www.tipografica.io)
}

